\title{
Evanescent excitation and collection of spontaneous Raman spectra using silicon nitride nanophotonic waveguides
}

\author{
Ashim Dhakal ${ }^{1,2 *}$, Ananth Z. Subramanian ${ }^{1,2}$, Pieter Wuytens ${ }^{1,2,3}$, Frédéric Peyskens ${ }^{1,2}$, Nicolas Le \\ Thomas $^{1,2}$ and Roel Baets ${ }^{1,2}$ \\ ${ }^{1}$ Photonics Research Group, INTEC Department, Ghent University-imec, Belgium \\ ${ }^{2}$ Center for Nano- and Biophotonics, Ghent University, Belgium \\ ${ }^{3}$ Department of Molecular Biotechnology, Ghent University-imec, Belgium \\ *Corresponding author: ashim.dhakal@intec.ugent.be
}

Received April 28, 2014

\begin{abstract}
We experimentally demonstrate the use of high contrast, CMOS-compatible integrated photonic waveguides for Raman spectroscopy. We also derive the dependence of collected Raman power with the waveguide parameters and experimentally verify the derived relations. Isopropyl alcohol (IPA) is evanescently excited and detected using single-mode silicon-nitride strip waveguides. We analyze the measured signal strength of pure IPA corresponding to $819 \mathrm{~cm}^{-1}$ Raman peak due to inphase C-C-O stretch vibration for several waveguide lengths, and deduce a pump power to Raman signal conversion efficiency on the waveguide to be at least $10^{-11}$ per $\mathrm{cm}$.
\end{abstract}

OCIS Codes: (130.0130) Integrated optics; (300.6450) Raman spectroscopy; (230.7380) Waveguides, channeled; (130.6010) Integrated Optics, Sensors; (290.5860) Raman scattering; (280.0280) Optical sensing and sensors

http://dx.doi.org/10.1364/OL.99.09999

Raman spectroscopy is a key technique for the detection and analysis of trace substances in applications ranging from physics and chemistry to biology and environmental sciences. However, Raman spectroscopic techniques often require bulky and/or expensive instrumentation to overcome the inherently weak nature of the Raman scattering process [1]. Typical spontaneous Raman spectroscopic systems use a microscopy system or a free space beam. These systems suffer from a poor conversion efficiency (defined as the signal to pump power ratio), mainly due to small interaction volume and poor efficiency of signal collection. To overcome the difficulties with these systems, waveguide-based systems have been demonstrated in the form of hollow fibers for Raman spectroscopy of gases [2] or liquid core waveguides for liquids [3]. In this letter, we demonstrate the use of integrated singlemode silicon nitride $\left(\mathrm{Si}_{3} \mathrm{~N}_{4}\right)$ waveguides [4] for excitation, collection, and enhancement of the Raman signal using the evanescent field of the guided mode. We envision that using mature photonic integration technologies [5-6] a complete Raman spectroscopic system can be integrated on a CMOScompatible chip. In addition to the reduction of cost and size of the instrumentation, the reduced mode area due to the use of high contrast nanophotonic waveguides also boosts the Raman signal. Based on our theoretical calculations [7-8], the use of a singlemode high contrast waveguide enhances the Raman signal by at least a factor of 500 per $\mathrm{cm}$ of waveguide length compared to the confocal microscopy systems. The use of cavities and nanoplasmonic structures defined on the waveguides [9] can further enhance the signal significantly. In this letter, we outline the theory of using the evanescent field of single mode photonic waveguides for Raman sensing and report the first experimental demonstration thereof.

An emitting molecule can be modelled as a dipole oscillating at a frequency $c / \lambda_{0}$. We consider that the molecule is located at the position $\boldsymbol{r}_{0}$ in the neighborhood of a photonic waveguide. Using Fermi's golden rule in a semi classical approach, the power coupled to a waveguide mode from the dipole can be calculated to be [10]:

$$
\frac{P_{w g}\left(\vec{r}_{0}\right)}{P_{0}}=\frac{3}{4 \pi} \frac{n_{g}}{n}\left(\frac{\lambda_{0}}{n}\right)^{2} \frac{\varepsilon\left(\vec{r}_{0}\right)\left|\hat{d}_{0} \cdot \vec{E}\left(\vec{r}_{0}\right)\right|^{2}}{\iint \varepsilon_{0} \varepsilon(\vec{r})|\vec{E}(\vec{r})|^{2} d \vec{r}}
$$

where $n_{g}$ is the group index of the mode, $n$ the refractive index at the location of the molecule, $\varepsilon(\boldsymbol{r})$ is the relative permittivity, $\hat{d}_{0}$ is the unit vector along the dipole, and $\mathrm{E}$ is the electric field strength of the mode. $P_{0}=\omega^{4}\left|\boldsymbol{d}_{0}\right|^{2} /\left(12 \pi \varepsilon_{0} c^{3}\right)$ is the power radiated by the dipole with dipole strength $\boldsymbol{d}_{\boldsymbol{0}}$ in free space. Now we assume that the dipole has a scalar polarizability $\alpha$, and is excited by a pump travelling in the same waveguide with power $P_{\text {pump }}$ and a frequency sufficiently near to $c / \lambda_{0}$. Then, using the normalization of the guided mode and its power [11],

$$
\begin{aligned}
& \left|d_{0}\right|^{2}=\alpha^{2}\left|E\left(\vec{r}_{0}\right)\right|^{2} \frac{n_{g} P_{\text {pump }}}{\iint c \varepsilon_{0} \varepsilon(\vec{r})|E(\vec{r})|^{2} d \vec{r}} ; \text { hence, } \\
& \frac{P_{\text {wg }}\left(r_{0}\right)}{P_{\text {pump }}}=\frac{\pi^{2}}{n}\left(\frac{\left|E\left(\vec{r}_{0}\right)\right|^{2}}{\iint \varepsilon_{0} \varepsilon(\vec{r})|E(\vec{r})|^{2} d \vec{r}} \frac{\alpha n_{g}}{\lambda_{0}}\right)^{2} \equiv \eta\left(r_{0}\right) \sigma
\end{aligned}
$$


where we have used the quadratic relationship between the polarizability and the scattering cross section $\sigma$ [1]. Note that Eq. (2) describes the overall efficiency, as a combined efficiency of excitation and collection, whereby waveguide enhancement effects [7-8] are taken into account. To arrive at Eq. (2), we have neglected the Stokes shift between the pump and the Raman signal. This has no significant impact on the analysis and conclusions that follow.

We now assume that the scattering molecules are suspended uniformly in the upper cladding of the waveguides with a density $\rho$. For emitters forming a thin sheet of thickness $d z$ orthogonal to the waveguide and covering the entire cladding area (see Fig. 1), the efficiency is given by the sum of the individual contributions:

$\frac{d P_{w g}}{P_{\text {pump }}}=d z \rho \sigma \iint_{\text {clad }} \eta(\vec{r}) d \vec{r}=d z \rho \sigma \eta_{0}$

where $\eta=\iint \eta(\boldsymbol{r}) d \boldsymbol{r}$ is the specific conversion efficiency of the waveguide. This unitless parameter depends only on the electromagnetic conditions and the geometry of the waveguide, which can be solved using mode solvers. Using the COMSOL finite element mode solver, the value of $\eta_{0}$ for the $\mathrm{Si}_{3} \mathrm{~N}_{4}$ strip waveguides used for the experiments [4] in this letter was estimated to be $6.4 \times 10^{-2}$ for a $785 \mathrm{~nm}$ pump for the TE fundamental mode of the waveguide. This value leads to a conversion efficiency $\left(\rho \sigma \eta_{0}\right)$ of $4 \times 10$. $10 / \mathrm{cm}[8]$ for pure IPA. Here, we have taken a cross-section of $7.9 \times 10^{-31} \mathrm{~cm}^{2} / \mathrm{sr}^{-1}$ for the $819 \mathrm{~cm}^{-1}$ line due to C-C-O stretch vibration which is calculated using the value provided in [12] and using the $1 / \lambda_{0}^{4}$ dependence of the cross-section[1].

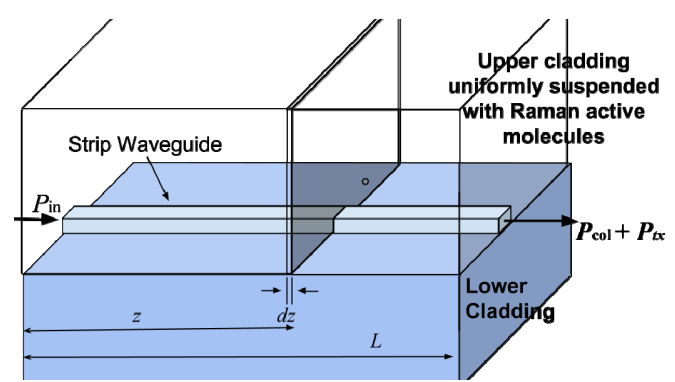

Fig. 1: Schematic of our analysis applied to the strip waveguide geometry. The particles are assumed to be embedded with a uniform density on the upper cladding. Symbols are explained in the text.

Although we have assumed that the entire upper cladding is covered by the Raman active molecules, a $350 \mathrm{~nm}$ thick layer of analyte is sufficient to produce an equivalent result, as the interaction takes place with exponentially decaying evanescent tail of the mode in the cladding with $1 / e$ length less than $250 \mathrm{~nm}$.

We now consider a practical situation, as depicted in Fig. 1. A pump laser with power $P_{\text {in }}$ is incident on the input facet of the waveguides, of which a fraction gets coupled to the waveguide mode with coupling efficiency $\gamma_{\text {in }}$ and propagates along the waveguide. During the propagation, molecules in the cladding are excited, and the scattered spontaneous Raman signal is collected via evanescent coupling to the same waveguide, as described by Eq. (3) in both forward and backward propagating directions. In the forward propagating configuration, only half of the collected power is measured at the output facet with an output coupling efficiency $\gamma_{\text {out }}$. Accounting for coupling losses and attenuation of the pump and collected signal in the waveguide, the ratio of signal power collected $\left(P_{c o}\right)$ at the end of the waveguide to the input power $\left(P_{i n}\right)$ is given by:

$$
\begin{aligned}
\frac{P_{c o l}(L)}{P_{\text {in }}} \equiv \xi(L) & =\rho \sigma \eta_{0} \gamma_{\text {in }} \gamma_{\text {out }} \int_{0}^{L} e^{-\alpha_{p} z} e^{-\alpha_{s}(L-z)} d z \\
& =\rho \sigma \eta_{0} \gamma_{\text {in }} \gamma_{\text {out }} e^{-\alpha_{p} L}\left[\frac{e^{\Delta \alpha L}-1}{\Delta \alpha}\right]
\end{aligned}
$$

where $\alpha_{p}$, and $\alpha_{s}$ are the waveguide losses at the pump wavelength and at the Stokes wavelength respectively and $\Delta \alpha=\alpha_{p}-\alpha_{s}$ is their difference. The term in brackets evolves to $L$ when $\Delta \alpha \rightarrow 0$.

If we assume that the coupling efficiencies from the waveguide end to the detector are equal for the pump and the Stokes signal, the Raman power collected at the end of the waveguide can be expressed in terms of the transmitted pump power $\left(P_{t}\right)$ at the detector, which can be directly measured. Thus, for many practical cases, it is convenient to define a quantity $\zeta(L)$ that is independent of coupling and waveguide losses:

$$
\zeta(L) \equiv \frac{P_{c o l}(L)}{P_{t x}(L)}=\rho \sigma \eta_{0}\left[\frac{e^{\Delta \alpha L}-1}{\Delta \alpha}\right]
$$

Along with Eq (2), Eq. (4) and (5) are the main theoretical results of this letter and express how the collected signal power depends on the input or transmitted pump power. In many situations, the quantity $\xi(L)$ is of practical interest, as the direct measure of the collected power for a given input power. However, it depends on coupling efficiencies and waveguide loss, which vary in the experiments. The quantity $\zeta(L)$ is independent of the coupling and the waveguide loss and can be used for purposes of direct analysis of signal strength with respect to concentration, cross-section, waveguide geometry and length. We will use both of these quantities to experimentally investigate the dependence of the Raman signal with the waveguide length.

The experimental setup is illustrated in Fig 2. A tunable Ti-Sapphire CW laser was used as the pump source. For the experiments, a wavelength of $785 \mathrm{~nm}$ is coupled to the waveguide by end-fire coupling using an aspheric lens of effective focal length $8 \mathrm{~mm}(\mathrm{NA}=0.5)$. The Raman signal (Stokes) co-propagating with the pump that is collected by the waveguide is then collimated via an achromat objective (50x, NA=0.9) towards an edge filter, with the 
edge wavelength at $795 \mathrm{~nm}$ to block pump light going into the spectrometer. The Raman signal is then focused to a single-mode optical fibre (cutoff $=770 \mathrm{~nm}$ ) using a parabolic mirror of $15 \mathrm{~mm}$ effective focal length $(\mathrm{NA}=0.2)$ and measured using a commercial spectrometer (AvaSpec-ULS2048XL).

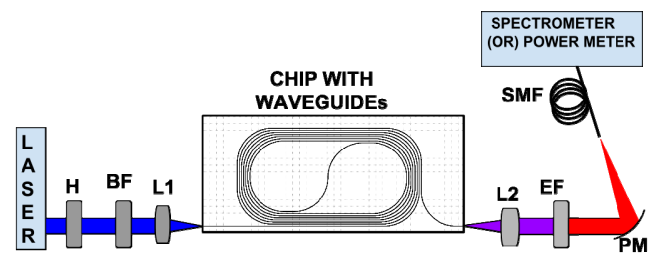

Fig 2: The schematic of experimental setup. $H$ : half-waveplate, $B F$ : Band-pass filter, L1: Aspheric lens. L2. Objective, EF: Long pass edge filter, $P M$. parabolic mirror, $S M F$. single-mode fibre

We use a $\mathrm{Si}_{3} \mathrm{~N}_{4}$ waveguide (cross section: $700 \mathrm{~nm}$ x 220 $\mathrm{nm}$ ) [4] on top of a $2.4 \mu \mathrm{m}$ silica cladding on a silicon substrate as our sensing platform. Several waveguides of lengths $0.7 \mathrm{~cm}, 1.6 \mathrm{~cm}, 2.5 \mathrm{~cm}, 4.4 \mathrm{~cm}$ and $8.1 \mathrm{~cm}$ wound up to form spirals (typical physical widths of the spiral is $200 \mu \mathrm{m}$ and the length of the spiral is about $625 \mu \mathrm{m}$ per $\mathrm{cm}$ of waveguide length, bend radii of $25,35,50,75$ and 100 $\mu \mathrm{m}$ were used, bend losses for all of the spirals are negligible) are used as the sensing region for the experiment. The laser power is set to $90 \mathrm{~mW}$, the polarization of the laser is set to couple into the TE mode of the waveguides. The laser is coupled to the waveguide with estimated total coupling loss from laser to detector (hence including both the in-coupling as well as the chipfibre coupling) of $14 \pm 2 \mathrm{~dB}$. Because of the edge filter, the pump transmission is measured and optimized at $800 \mathrm{~nm}$ wavelength which was verified to be practically the same as for the pump wavelength $785 \mathrm{~nm}$ without the filter.

A droplet of IPA is used as an analyte and is applied on top of the waveguides to cover the entire waveguide region. We ensure that IPA covers the entire waveguide by visually monitoring the chip with a camera on top. The spectra were recorded before and after application of IPA, as shown in Fig. 3. Before application of IPA we see a broad Raman emission in the range $2100-2400 \mathrm{~cm}^{-1}$ with a peak around $2330 \mathrm{~cm}^{-1}(960 \mathrm{~nm})$. Based on the measurements with a confocal Raman microscope operating with $532 \mathrm{~nm}$ pump, this peak is identified as Raman emission from the $\mathrm{Si}_{3} \mathrm{~N}_{4}$ material that forms the waveguide core. Another feature of the spectrum is the broad luminescence below $1200 \mathrm{~cm}^{-1}$ from the waveguide material. Immediately after application of IPA, the intense Raman peak at $819 \mathrm{~cm}^{-1}(839 \mathrm{~nm})$ due to the inphase C-C-O stretch vibration [13] is readily observed. The complete spectrum due to IPA is then extracted using the difference of the normalized spectrum before and after application of IPA. Since there is a difference in the modal properties of the waveguide before and after application of IPA, simply subtracting the spectra will not yield the desired spectrum of IPA. First, there is an increase in the transmitted pump and signal power after application of IPA. This is due to reduced sidewall scattering contribution to the waveguide losses because the cladding index has significantly changed (refractive index of IPA $=1.377$ ). Second, the confinement of the mode in the waveguide core and cladding changes, leading to a modified background contribution from the corresponding materials. To account for the change in the intensity due to decreased waveguide losses, we normalize the two spectra with the respective intensities corresponding to the peak due to $\mathrm{Si}_{3} \mathrm{~N}_{4}$ at $2330 \mathrm{~cm}^{-1}$. This also ensures that the invariant part of the background contributions from the waveguide core measured before application of IPA is removed when we subtract the normalized spectra before and after application of IPA. The residual background due to the changes in the cladding conditions is removed by fitting the remainder spectrum with truncated polynomials [14]. The resultant spectrum is then converted back to original units using the corresponding counts of the $2330 \mathrm{~cm}^{-1}$ peak used for normalization. The resultant spectrum, as we can see in Fig. 3 b), matches with the spectrum of IPA measured independently [15].

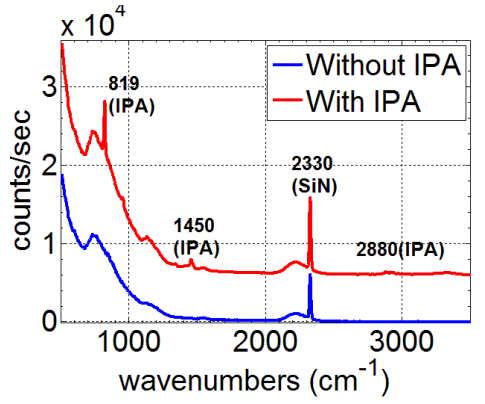

a)

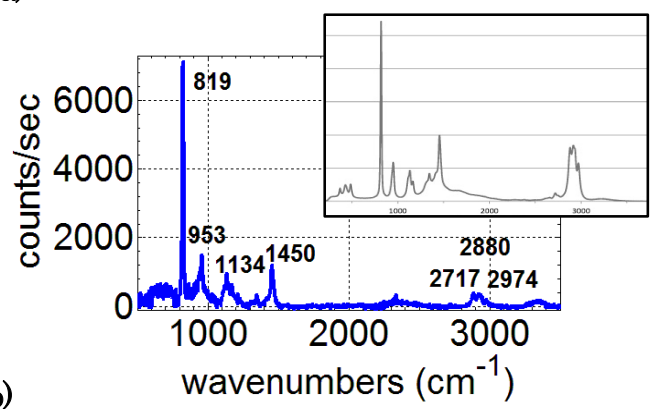

Fig 3: a) Raw spectrum measured from a $1.6 \mathrm{~cm}$ waveguide without IPA (blue) and with IPA (red). The spectra are shifted vertically for clarity with respective zeros at $3500 \mathrm{~cm}^{-1}$. b) The spectrum of IPA extracted from the spectra in Fig 3(a). The spectrum measured independently using a commercial spectrometer is also shown in the inset (from [15]).

Fig. 4 a) shows the measured $\xi(L)$ for the $817 \mathrm{~cm}^{-1}$ peak due to the IPA for several waveguide lengths. Ten different sets of waveguide samples with different lengths were used for the measurements. The average and standard deviation of the measured $\xi(L)$ and the curve for Eq. (4) that fits the averages with the least squared error is also shown as a guide to the eye. The results follow the trend described by Eq. (4), in spite of considerable standard deviation due to differences in the quality of the 
waveguide facets, the particles stuck on the waveguides during cleaving and imperfections in waveguide processing. The waveguide loss calculated from the transmission data using cutback method, $2.1 \pm 0.4 \mathrm{~dB} / \mathrm{cm}$ is very close to the value $2.2 \mathrm{~dB} / \mathrm{cm}$ obtained from the least square fit of Eq. (4). We see that the optimal waveguide length for $\xi(L)$ is given by $L=1 / \alpha$, as can be inferred from Eq. (4)

a)

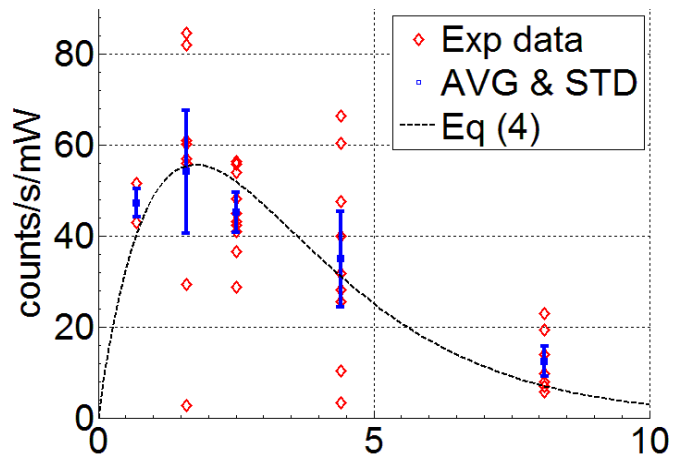
waveguide length $(\mathrm{cm})$

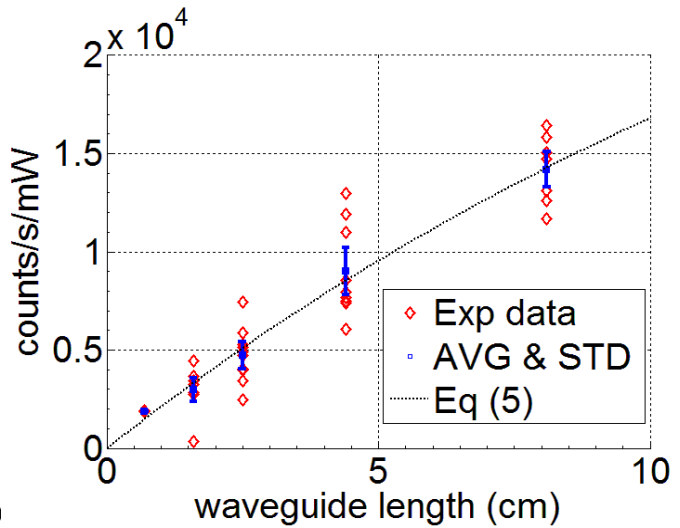

Fig 4: a) $\xi(L)$ and b) $\zeta(L)$ for $819 \mathrm{~cm}^{-1}$ peak due to IPA measured using photonic waveguides of several lengths. Red diamond markers: actual measured data, Blue solid square with error bars: mean and standard deviation, Black dashed line: theoretical fit, to respective equations as a guide for the eye.

In Fig. 4 (b) we show the measured $\zeta(L)$ for the IPA peak at $819 \mathrm{~cm}^{-1}$. As expected from $\mathrm{Eq}(5)$, for $\Delta \alpha \approx 0$, we see a quasi-linear trend. Using the least squared error fit, the value of $\rho \sigma \eta_{0}$ for the IPA-waveguide system was extracted to be 2200 counts/s per $\mathrm{mW}$ of transmitted pump power per $\mathrm{cm}$ waveguide. $\Delta \alpha$ was extracted to be $0.2 \mathrm{~dB} / \mathrm{cm}$, which is equal to the measured value within the limits of experimental errors. As seen in Fig. 4, unlike the parameter $\xi(L)$, which depends on coupling and waveguide losses hence suffers from a large variance, $\zeta(L)$ has smaller variance and is suitable for directly assessing the specific efficiency of Raman scattering with the waveguide mode as defined by Eq. (5).

Using the measured sensitivity of the spectrometer in our configuration (in the order of 100 counts $/ \mathrm{ms} / \mathrm{pW}$ ), the value of $\rho \sigma \eta_{0}$ can be calculated to be in the order of $10^{-}$ $11 / \mathrm{cm}$. Thus, the measured value of conversion efficiency $\rho \sigma \eta_{0}$ is about one order of magnitude lower than the value estimated in the theoretical section. This is a reasonable correspondence given the combination of uncertainties on the scattering cross-section and the measurement error.

To conclude, we have experimentally demonstrated Raman spectroscopy on a chip using single mode integrated optical waveguides, by exploiting enhanced light scattering due to the strong confinement in high index contrast waveguides as well as the long interaction length of the waveguide mode with the analyte in the cladding. Further, we have developed a theory for excitation and collection of spontaneous Raman signal using the photonic waveguides and identified the relevant parameters of interest $\eta_{0,} \xi(L)$ and $\zeta(L)$. For a given pump power within a typical single mode nanophotonic $\mathrm{Si}_{3} \mathrm{~N}_{4}$ waveguide discussed in [4], we report a conversion efficiency in the order of $10^{-11} / \mathrm{cm}$ for the $819 \mathrm{~cm}^{-1}$ Raman line of IPA.

The authors acknowledge the ERC advanced grant InSpectra for partial funding and imec, Leuven for processing of the waveguides

\section{References.}

1. D. A. Long, The Raman Effect: A Unified Treatment of the Theory of Raman Scattering by Molecules, J Wiley \& Sons, Ltd, Chichester, UK (2002)

2. M. P. Buric, K. P. Chen, J. Falk, and S. D. Woodruff, Appl. Opt. 47, 23 (2008)

3. J.S.W. Mak, S.Rutledge, R.M. Abu-Ghazalah, F. Eftekhari, J. Irizar, N.C.M. Tam, G. Zheng, A. S. Helmy, Prog. Quant. Elec. 37 (2013)

4. A. Z. Subramanian, P. Neutens, A. Dhakal, R. Jansen, T. Claes, X. Rottenberg, F. Peyskens, S. Selvaraja, P. Helin, B. Du Bois, K. Leyssens, S. Severi, P. Deshpande, R. Baets, P. Van Dorpe, IEEE Photonics J., 5, 2202809 (2013)

5. D. Dai, J. Bauters, and J.E. Bowers, Light: Sci. Appl. 1, 1 (2012)

6. M. Pollnau, N. Ismail, B.I. Akca, K. Wörhoff, R.M. de Ridder; Proc. SPIE 8266, Silicon Photonics VII (2012)

7. A. Dhakal, A.Z. Subramanian, N. Le Thomas, R. Baets, 16th European Conf. on Integrated Optics, Spain (2012)

8. A. Dhakal, F. Peyskens, A.Z. Subramanian, N. Le Thomas, R. Baets, Advanced Photonics Congress, PR, USA (2013)

9. F. Peyskens, A.Z. Subramanian, A. Dhakal, N. Le Thomas, R. Baets, CLEO, USA (2013)

10. Y.C. Jun, R.M. Briggs, H. A. Atwater, M.L. Brongersma, Opt. Express, 17, 7479 (2009).

11. A.W. Snyder, J.D. Love, Optical Waveguide Theory, Chapman and Hall, New York, (1983).

12. M.J. Colles, J.E Griffiths. J. Chem. Phys., 7, 56, (2003).

13. D Lin-Vien; N. B. Colthup; W. G. Fateley, J. G. Grasselli, The handbook of infrared and Raman characteristic frequencies of Organic Molecules, Academic Press, CA, (1991)

14. V. Mazet, C. Carteret, D. Brie, J. Idier, B. Humbert. Chemom. Intell. Lab. Syst. 76, 2 (2005)

15. http://www.oceanoptics.com/images/spectraisopropanol.jpg 


\section{References with title.}

1. D. A. Long, "The Raman Effect: A Unified Treatment of the Theory of Raman Scattering by Molecules', John Wiley \& Sons, Ltd, Chichester, UK (2002)

2. M. P. Buric, K. P. Chen, J. Falk, and S. D. Woodruff, 'Enhanced spontaneous Raman scattering and gas composition analysis using a photonic crystal fiber', Appl. Opt. 47, 23 (2008)

3. J.S.W. Mak, S.Rutledge, R.M. Abu-Ghazalah, F. Eftekhari, J. Irizar, N.C.M. Tam, G. Zheng, A. S. Helmy, 'Recent developments in optofluidic-assisted Raman spectroscopy', Prog. Quant. Elec. 371 (2013)

4. A. Z. Subramanian, P. Neutens, A. Dhakal, R. Jansen, T. Claes, X. Rottenberg, F. Peyskens, S. Selvaraja, P. Helin, B. Du Bois, K. Leyssens, S. Severi, P. Deshpande, R. Baets, P. Van Dorpe, 'Low-Loss Singlemode PECVD Silicon Nitride Photonic Wire Waveguides for 532-900 nm Wavelength Window Fabricated Within a CMOS Pilot Line'. IEEE Photonics J., 5, 2202809 (2013)

5. D. Dai, J. Bauters, and J.E. Bowers, 'Passive technologies for future large-scale photonic integrated circuits on silicon: polarization handling, light nonreciprocity and loss reduction', Light: Sci. Appl. 1, 1 (2012)

6. M. Pollnau, N. Ismail, B.I. Akca, K. Wörhoff, R.M. de Ridder, 'Biophotonic sensors on a silicon chip for Raman spectroscopy and optical coherence tomography', Proc. SPIE 8266, Silicon Photonics VII (2012)

7. A. Dhakal, A.Z. Subramanian, N. Le Thomas, R. Baets, "The role of index contrast in the efficiency of absoprtion and emission of a luminescent particle near a slab waveguide', 16th European Conf. on Integrated Optics, Spain (2012)

8. A. Dhakal, F. Peyskens, A.Z. Subramanian, N. Le Thomas, R. Baets, 'Enhancement of light absorption, scattering and emission in high index contrast waveguides', Advanced Photonics Congress, PR, USA (2013)

9. F. Peyskens, A.Z. Subramanian, A. Dhakal, N. Le Thomas, R. Baets, 'Enhancement of Raman Scattering Efficiency by a Metallic Nano-antenna on Top of a High Index Contrast Waveguide', CLEO, USA (2013)

10. Y.C. Jun, R.M. Briggs, H. A. Atwater, M.L. Brongersma, 'Broadband enhancement of light emission in silicon slot waveguides', Opt. Express, 17, 7479 (2009).

11. A.W. Snyder, J.D. Love, 'Optical Waveguide Theory', Chapman and Hall, New York, (1983).

12. M.J. Colles, J.E Griffiths, 'Relative and absolute Raman scattering cross sections in liquids', J. Chem. Phys., 7, 56, (2003).

13. D Lin-Vien; N. B. Colthup; W. G. Fateley, J. G. Grasselli, "The handbook of infrared and Raman characteristic frequencies of Organic Molecules' Academic Press, CA, pp 45-59 (1991)

14. V. Mazet, C. Carteret, D. Brie, J. Idier, B. Humbert, 'Background removal from spectra by designing and minimising a non-quadratic cost function', Chemom. Intell. Lab. Syst. 76, 2 (2005)

15. http://www.oceanoptics.com/images/spectraisopropanol.jpg 